\title{
Development of an LCD-Photomask-Based Desktop Manufacturing System
}

\author{
Ren C. Luo, Fellow, IEEE, and Jyh Hwa Tzou
}

\begin{abstract}
A liquid crystal display (LCD)-photomask-based desktop manufacturing system that includes software and hardware configuration is described. The software design includes a slicing algorithm, an LCD photomask display, a process-user interface, and a motion control program. The bucket-sorting algorithm is used in the slicing preprocessing for search speed enhancement. The slicing time ratio can be reduced to nearly $25 \%$ with five buckets. The hardware configuration of this architecture includes an LCD photomask, an optical system, a $z$-axis elevator, and a personal-computer-based control system. The matrix optics assists the optical system design of the proposed RP machine. After the slicing process, a cross-sectional contour of each layer is transferred to the LCD photomask. The optical system emits parallel light upward through the photomask to expose and solidify the entire layer at once. This visible light can expose and solidify an entire layer at once, layer by layer, until the whole part is finished. We have physically demonstrated the complete system, including hardware and software implementation, and the experimental results are as what was expected and are described here.
\end{abstract}

Index Terms-Bucket sorting, desktop manufacturing system, liquid crystal display (LCD) photomask, matrix optics.

\section{INTRODUCTION}

D ESKTOP manufacturing refers to various rapid prototyping (RP) techniques, where 3-D components are directly built, layer by layer, from a computer data description or a computer-aided design (CAD) file. Due to their ability and the relative ease of transforming a conceptual design into a physical model, desktop manufacturing technologies have met escalating demand in the industry for shortening new product development cycle time. The layer-by-layer fabrication methodology also allows complex models to be made with ease.

In desktop manufacturing processes, the geometry of the object that will be manufactured can be obtained from CAD model data, an existing object (through reverse engineering) [1]-[3], or mathematical data (e.g., surface equations) [4]. Prashant $e t$ al. [5] reviewed many process planning techniques in layer manufacturing. Most desktop manufacturing systems accept model data that are described in an intermediate file format called (stereolithography) STL. This file format approximates the original model geometry by using a series of triangular facets.

Manuscript received June 11, 2004; revised March 5, 2008. Current version published October 1, 2008

R. C. Luo is with the Department of Electrical Engineering, National Taiwan University, Taipei 10617, Taiwan, R.O.C. (e-mail: renluo@ntu.edu.tw).

J. H. Tzou is with the Department of Mechanical Engineering, Wu-Feng Institute of Technology, Chia-Yi 621, Taiwan, R.O.C. (e-mail: tzoujyh@mail. wfc.edu.tw).

Color versions of one or more of the figures in this paper are available online at http://ieeexplore.ieee.org.

Digital Object Identifier 10.1109/TIE.2008.922603
After loading an STL model, a slicing procedure is, then, applied to the tessellated model. In this process, the model is intersected with a set of horizontal planes to create a series of cross sections or slices that comprise contours that represent the material boundaries of the part that will be generated. The contours are subsequently used to generate the numerically controlled tool paths for the desktop manufacturing system.

There are many commercial RP systems that are currently available on the market, such as InVision [6], Objet [7], Perfactory [8], stereolithography apparatus (SLA) [9], and fused deposition modeling (FDM) [10]. The InVision 3-D printer combines 3-D Systems' multijet modeling printing technology with an acrylic photopolymer model material. Objet's Polyjet technology works by jetting photopolymer materials in ultrathin (i.e., $0.016 \mathrm{~mm}$ ) layers onto a build tray, layer by layer, until the part is complete. The Perfactory RP system uses a photomonomer resin and a digital light processing (DLP) projector for polymerizing 3-D finished parts. SLA and FDM are old RP processes. Both the laser beam of the SLA system and the thermal extrusion head of the FDM system generate 2-D crosssectional areas by using 1-D tool paths. The disadvantages of these systems are lower speed, the requirement for an expensive $x y z$ table, and three-axis motion control system. Another old RP process, i.e., solid ground curing (SGC) [11], involves creating a temporary photomask of each layer, applying a thin coating of photopolymers, and exposing the layer to a burst of ultraviolet light for curing it. Because the cross sections of one layer are simultaneously cured, the SGC system has a faster build speed. However, the mechanism of the SGC system is complex, and the price is very expensive.

The purpose of this paper is to develop a low-cost desktop manufacturing system. We use a liquid crystal display (LCD) panel as a photomask [12]. With the bottom exposure method, the image of the LCD photomask is calculated from the sliced data. The desktop manufacturing system has the advantages of low cost, compactness, and no special physical support requirement, making it suitable for use in offices.

Ventura et al. [13] developed a direct photo shaping process for the layer-by-layer fabrication of functional ceramic components. Each layer is photoimaged by a LCD or a DLP projection system. Young et al. [14] described a novel device for producing 3-D objects, which has been developed using an LCD as a programmable dynamic mask and visible light for initiating photopolymerization. Monneret et al. [15] presented a new process of microstereolithography for manufacturing freeform solid 3-D microcomponents, with its outer dimensions being in the millimeter size range. Huang and Jiang [16] analyzed the shrinkage deformation of the mask-type stereolithography process. Jiang et al. [17] developed a masked 


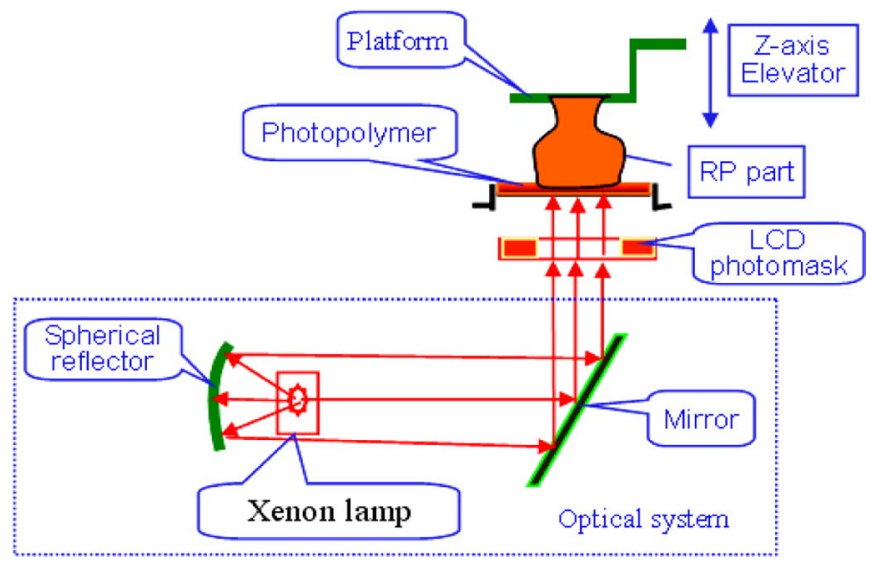

Fig. 1. Configuration of the LCD-photomask-based desktop manufacturing system.

photopolymerization RP system that uses an LCD panel as a dynamic mask with an upper exposure skill.

\section{LCD-PHOTOMASK-BASED DESKTOP MANUFACTURING SYSTEM}

The LCD-photomask-based desktop manufacturing system structure is illustrated in Fig. 1. The hardware configuration of this system includes an LCD photomask, an optical system, a $z$-axis elevator, and a personal computer (PC)-based control system. The optical system can generate parallel light that passes through the LCD photomask to cure the photopolymer. The RP part is generated layer by layer and is attached to a platform that rises as each successive layer is attached to the lowermost face. The resin is deposited onto the transparent bottom plate. The platform and the previously built structure are lowered into the resin, leaving a liquid film between the part and the bottom plate, with a correct thickness for the next layer. The new layer is formed beneath the platform by exposing the LCD photomask. After the layer is finished, the platform is raised, separating the layer from the bottom plate, filling and wiping the resin, and repeating the process until all layers are fabricated. The completed RP part is, then, removed from the platform, post cured, and finished, if needed.

The desktop manufacturing system architecture is illustrated in Fig. 2. The $z$-axis elevator with a high-precision ball screw is driven by an alternating-current servomotor. A PC-based DSP motion controller is used to control the movement of the $z$-axis elevator. The LCD photomask is connected to the video graphics array card of the computer.

The architecture of the proposed system includes five main components: 1) a data processing unit; 2) an LCD photomask; 3) an optical system; 4) a PC-based DSP motion control system; and 5) a $z$-axis elevator. The details of these components are discussed as follows.

\section{A. Data Processing Unit}

The data processing unit performs the slicing procedure and the photomask generation process. The slicing procedure transforms the 3-D CAD model into a set of 2-D layer contours. According to this contour data, the photomask generation program exports the contour of each layer to the LCD photomask.

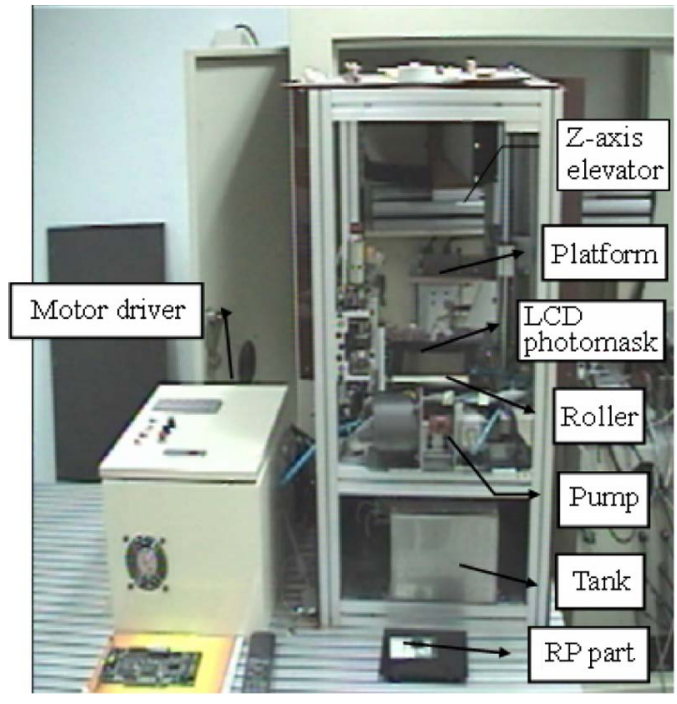

Fig. 2. Photo of the LCD-photomask-based desktop manufacturing system.

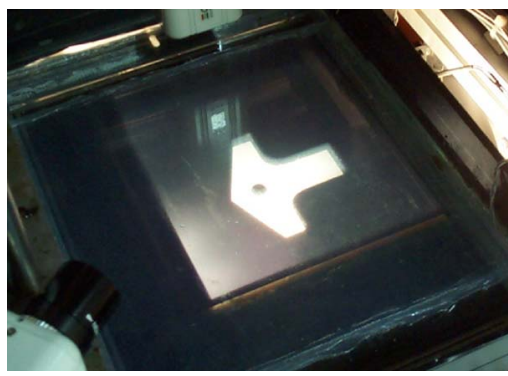

Fig. 3. LCD photomask of the proposed system

Note that the region inside the contour is displayed in white and the region outside the contour is displayed in black.

\section{B. LCD Photomask}

An LCD serves as a photomask that is used to display layer contours. The light source emits parallel light upward through the transparent portions of the LCD mask to expose and solidify the entire layer at once. As shown in Fig. 3, a 14.1-in thin-film transistor LCD with $1024 \times 768$ pixels is used here. Each pixel is $0.28 \mathrm{~mm}$ in both width and length, yielding a photomask resolution of under $0.28 \mathrm{~mm}$. An insulating membrane is located below the LCD photomask for insulating the resin from the ultraviolet rays and heat that the light source produced. Furthermore, the size of the RP part that can be produced in this system is restricted by the size of the LCD panel used. If we can use a larger LCD panel, the size of the RP part could be increased.

\section{Optical system}

The optical system strongly influences the system structure, forming method, and building time for parts. The proposed system uses a NAF-200N photo-curable liquid resin (from Denken Engineering Company Ltd., Oita, Japan) as the building material. NAF-200N solidifies under exposure to $680-\mathrm{nm}$ visible light. A $275-\mathrm{W}$ xenon lamp serves as the light source. The optical spectrum of this xenon lamp, which is detected by a spectrometer, is shown in Fig. 4, where the spectral radiance is observed with maximum power at a 736-nm wavelength and an adequate amount of energy at $680 \mathrm{~nm}$. The experimental results 


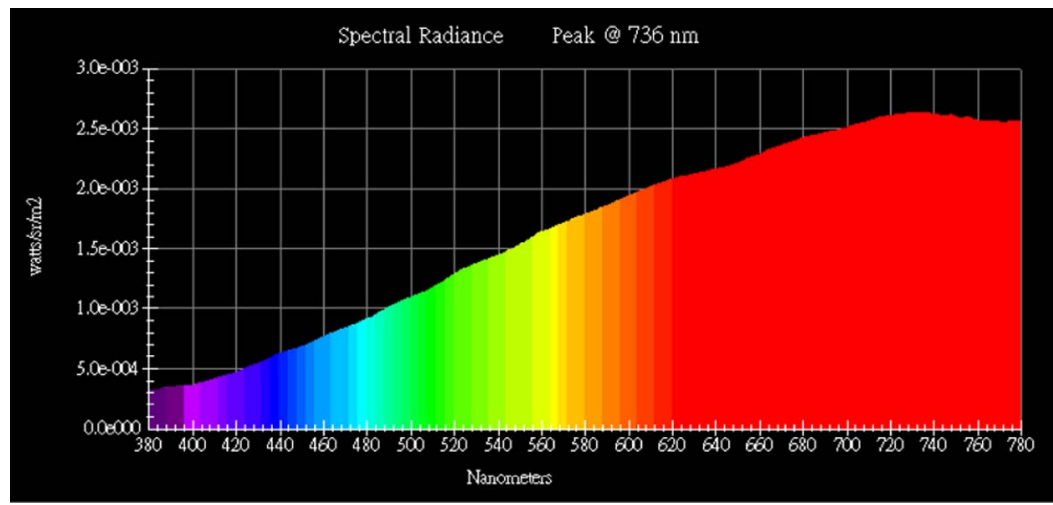

Fig. 4. Spectrogram of the 275-1W xenon lamp.

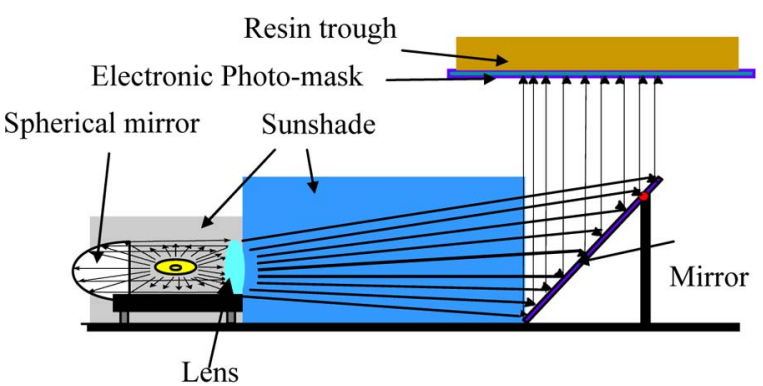

(a)

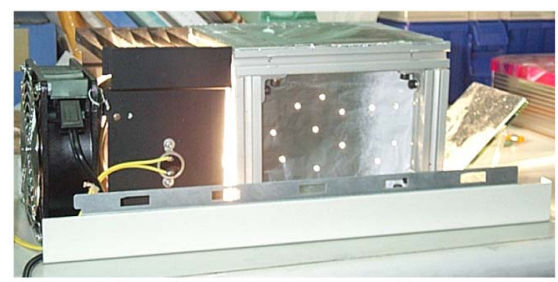

(b)

Fig. 5. (a) Design of the curing light source. (b) Real structure of the curing light source.

confirm the ready solidification of NAF-200N under exposure to this xenon lamp. According to the experimental results, the layer thickness for one layer is $0.254 \mathrm{~mm}$. The curing time for one layer is $135 \mathrm{~s}$.

The optical system design is illustrated in Fig. 5(a). The actual structure is shown in Fig. 5(b). The proposed system uses plane shaping instead of line shaping. The visible light source is emitted in a bottom-up manner, instead of a top-down way, to reduce the amount of wasted resin.

The ray tracing method is an important tool in geometrical optics. Matrix optics [18], [19] were used to design the RP machine optical system. A ray is described by its position and its angle with respect to the optical axis. The matrix form of several optical components can be shown as follows.

1) Free-Space Propagation: As shown in Fig. 6, a ray that traverses a distance $d$ is altered in accordance with $y_{2}=y_{1}+$ $\theta_{1} d$ and $\theta_{2}=\theta_{1}$. The ray-transfer matrix is

$$
T=\left[\begin{array}{ll}
1 & d \\
0 & 1
\end{array}\right]
$$

In addition

$$
\left[\begin{array}{l}
y_{2} \\
\theta_{2}
\end{array}\right]=\left[\begin{array}{ll}
1 & d \\
0 & 1
\end{array}\right]\left[\begin{array}{l}
y_{1} \\
\theta_{1}
\end{array}\right]=T\left[\begin{array}{l}
y_{1} \\
\theta_{1}
\end{array}\right]
$$

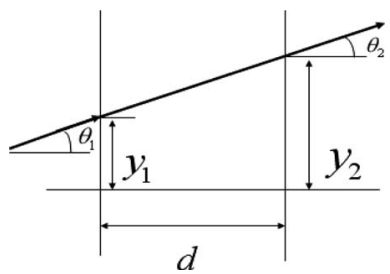

Fig. 6. Free-space propagation.

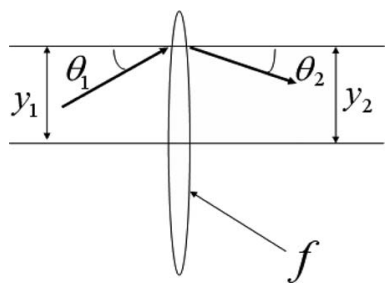

Fig. 7. Transmission through a thin lens.

2) Transmission Through a Thin Lens: As shown in Fig. 7, the relation between $\theta 1$ and $\theta 2$ for paraxial rays is transmitted through a thin lens of focal length $f$. Since the height remains unchanged (i.e., $y_{2}=y_{1}$ ), the refraction matrix of the thin lens is

$$
A=\left[\begin{array}{cc}
1 & 0 \\
-\frac{1}{f} & 1
\end{array}\right]
$$

In addition

$$
\left[\begin{array}{l}
y_{2} \\
\theta_{2}
\end{array}\right]=\left[\begin{array}{cc}
1 & 0 \\
-\frac{1}{f} & 1
\end{array}\right]\left[\begin{array}{l}
y_{1} \\
\theta_{1}
\end{array}\right]=A\left[\begin{array}{l}
y_{1} \\
\theta_{1}
\end{array}\right]
$$

3) Reflection From a Planar Mirror: As shown in Fig. 8, the ray position is not altered (i.e., $y_{2}=y_{1}$ ), and we conclude that $\theta_{2}=\theta_{1}$. The ray-transfer matrix is, therefore, the following identity matrix:

$$
R=\left[\begin{array}{ll}
1 & 0 \\
0 & 1
\end{array}\right]
$$

In addition

$$
\left[\begin{array}{l}
y_{2} \\
\theta_{2}
\end{array}\right]=\left[\begin{array}{ll}
1 & 0 \\
0 & 1
\end{array}\right]\left[\begin{array}{l}
y_{1} \\
\theta_{1}
\end{array}\right]=R\left[\begin{array}{l}
y_{1} \\
\theta_{1}
\end{array}\right]
$$




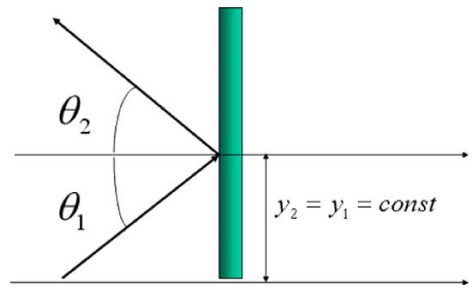

Fig. 8. Reflection from a planar mirror.

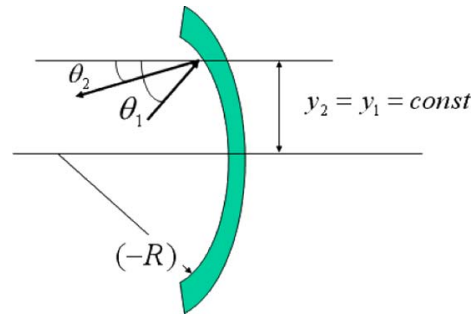

Fig. 9. Reflection from a spherical mirror

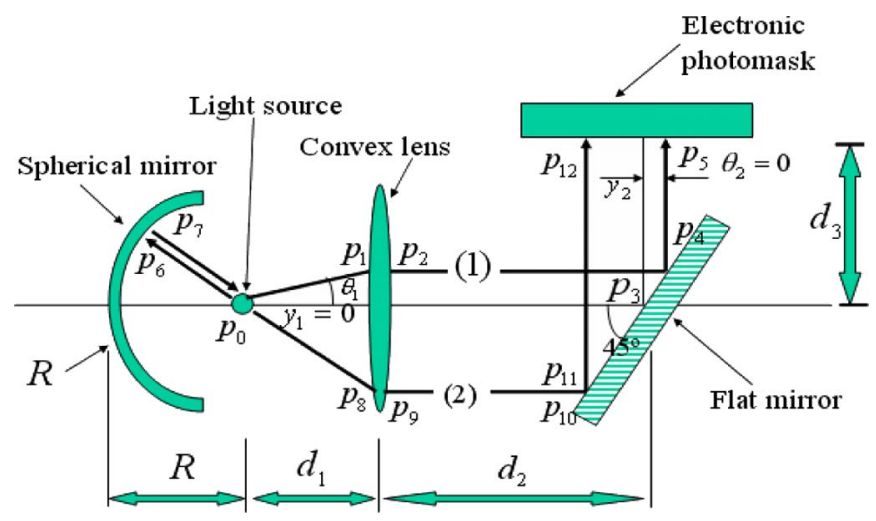

Fig. 10. Ray tracing diagram of the optical system of the RP machine.

4) Reflection From a Spherical Mirror: As shown in Fig. 9, the ray position is not altered (i.e., $y_{2}=y_{1}$ ). The reflection matrix of a spherical mirror is

$$
S=\left[\begin{array}{cc}
1 & 0 \\
-\frac{2}{R} & 1
\end{array}\right] .
$$

In addition

$$
\left[\begin{array}{l}
y_{2} \\
\theta_{2}
\end{array}\right]=\left[\begin{array}{cc}
1 & 0 \\
-\frac{2}{R} & 1
\end{array}\right]\left[\begin{array}{l}
y_{1} \\
\theta_{1}
\end{array}\right]=S\left[\begin{array}{l}
y_{1} \\
\theta_{1}
\end{array}\right]
$$

The ray tracing diagram of the optical system of the RP machine is shown in Fig. 10. The light source $\mathrm{P}_{0}$ (i.e., the xenon lamp) is located on the focus of the biconvex lens and at the center of the spherical mirror. The ray that is emitted by light source $\mathrm{P}_{0}$ can be divided into two parts: 1 ) ray trajectory 1 directly transmits through the thin lens and 2) ray trajectory 2 is reflected from the spherical mirror.

These two ray trajectories are discussed as follows:

\section{- Ray trajectory 1: $\mathbf{P}_{0} \mathbf{P}_{1} \mathbf{P}_{2} \mathbf{P}_{3} \mathbf{P}_{4} \mathbf{P}_{5}$.}

The ray that is emitted from light source $\mathrm{P}_{0}$ transmits through the thin lens. After reflecting the ray from the flat mirror, the reflected parallel light can be generated onto

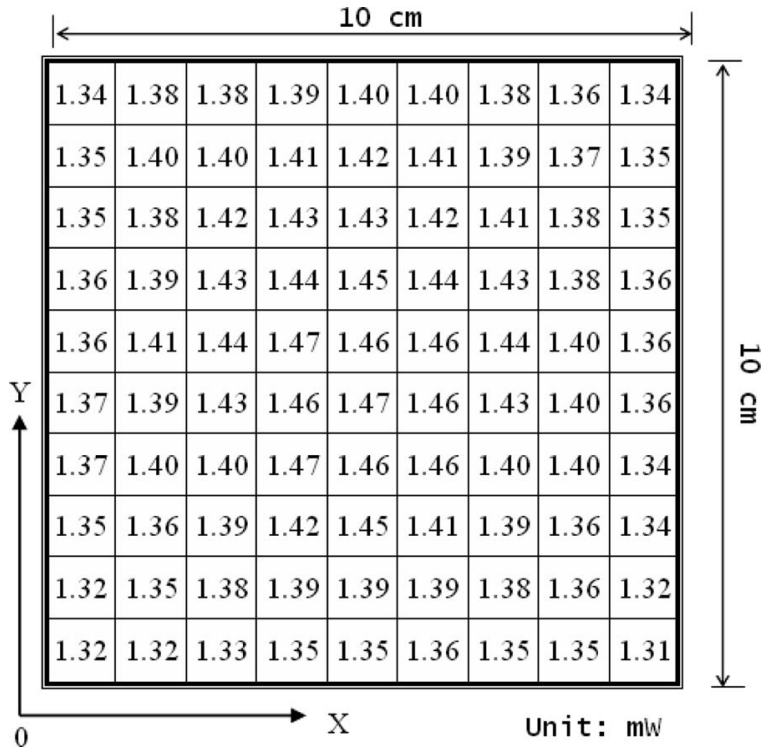

Fig. 11. Light power spreads on the LCD photomask.

the LCD photomask position (i.e., $\mathrm{P}_{5}$ ). The system matrix is, then, defined as

$$
\begin{aligned}
\boldsymbol{M}_{1} & =\boldsymbol{T}_{54} \boldsymbol{R}_{43} \boldsymbol{T}_{32} \boldsymbol{A}_{21} \boldsymbol{T}_{10} \\
& =\left[\begin{array}{cc}
1 & d_{3} \\
0 & 1
\end{array}\right]\left[\begin{array}{ll}
1 & 0 \\
0 & 1
\end{array}\right]\left[\begin{array}{cc}
1 & d_{2} \\
0 & 1
\end{array}\right]\left[\begin{array}{cc}
1 & 0 \\
-\frac{1}{f} & 1
\end{array}\right]\left[\begin{array}{cc}
1 & d_{1} \\
0 & 1
\end{array}\right] \\
& =\left[\begin{array}{cc}
1-\frac{d_{2}}{f}-\frac{d_{3}}{f} & d_{1}+d_{2}\left(-\frac{d_{1}}{f}+1\right)-\frac{d_{1} d_{3}}{f}+d_{3} \\
-\frac{1}{f} & -\frac{d_{1}}{f}+1
\end{array}\right]
\end{aligned}
$$

where

$T_{10} \quad$ ray-transfer matrix from $\mathrm{P}_{0}$ to $\mathrm{P}_{1}$;

$A_{21}$ thin-lens refraction matrix from $\mathrm{P}_{1}$ to $\mathrm{P}_{2}$;

$T_{32}$ ray-transfer matrix from $\mathrm{P}_{2}$ to $\mathrm{P}_{3}$;

$R_{43}$ reflection matrix of a planar mirror from $\mathrm{P}_{3}$ to $\mathrm{P}_{4}$;

$T_{54}$ ray-transfer matrix from $\mathrm{P}_{4}$ to $\mathrm{P}_{5}$.

Thus, the ray at point $\mathrm{P}_{5}$ on the LCD photomask position is given by

$$
\begin{aligned}
{\left[\begin{array}{l}
y_{2} \\
\theta_{2}
\end{array}\right]=} & \boldsymbol{M}_{1} *\left[\begin{array}{l}
y_{1} \\
\theta_{1}
\end{array}\right] \\
= & {\left[\begin{array}{cc}
1-\frac{d_{2}}{f}-\frac{d_{3}}{f} & d_{1}+d_{2}\left(-\frac{d_{1}}{f}+1\right)-\frac{d_{1} d_{3}}{f}+d_{3} \\
-\frac{1}{f} & -\frac{d_{1}}{f}+1
\end{array}\right] } \\
& \times\left[\begin{array}{l}
y_{1} \\
\theta_{1}
\end{array}\right] .
\end{aligned}
$$

If $d_{1}=\mathrm{f}$ and $y_{1}=0$, (6) can be simplified as

$$
\left[\begin{array}{l}
y_{2} \\
\theta_{2}
\end{array}\right]=\left[\begin{array}{cc}
1-\frac{d_{2}}{f}-\frac{d_{3}}{f} & f \\
-\frac{1}{f} & 0
\end{array}\right]\left[\begin{array}{c}
0 \\
\theta_{1}
\end{array}\right] .
$$

Consequently

$$
y_{2}=f \theta_{1} \quad \theta_{2}=0 .
$$

- Ray trajectory 2: $\mathrm{P}_{0} \mathrm{P}_{6} \mathrm{P}_{7} \mathrm{P}_{8} \mathrm{P}_{9} \mathrm{P}_{10} \mathrm{P}_{11} \mathrm{P}_{12}$.

As shown in Fig. 10, the ray that is emitted from light source $\mathrm{P}_{0}$ is reflected by the spherical mirror. After the 


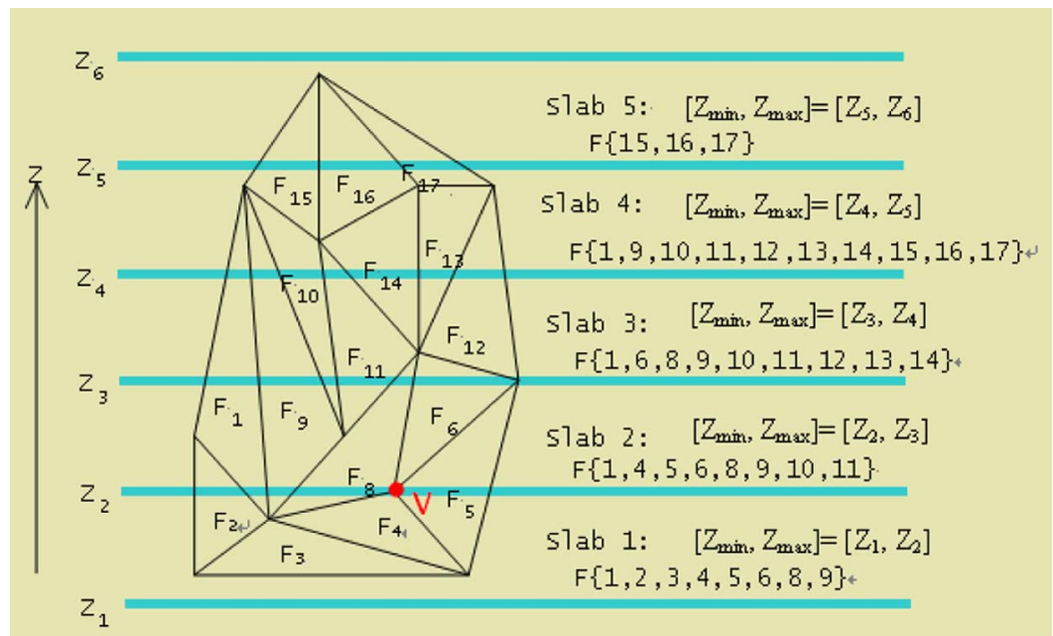

Fig. 12. Bucket-sorting algorithm for data sorting,

reflected ray is transferred through the thin lens and flat mirror, the parallel light can be generated onto the LCD photomask position (i.e., $\mathrm{P}_{12}$ ). The system matrix is then defined as

$$
\begin{aligned}
\boldsymbol{M}_{2}= & \boldsymbol{T}_{12} \cdot{ }_{11} \boldsymbol{R}_{11} \cdot{ }_{10} \boldsymbol{T}_{10} \cdot{ }_{9} \boldsymbol{A}_{98} \boldsymbol{T}_{87} \boldsymbol{S}_{76} \boldsymbol{T}_{60} \\
= & {\left[\begin{array}{cc}
1 & d_{3} \\
0 & 1
\end{array}\right]\left[\begin{array}{cc}
1 & 0 \\
0 & 1
\end{array}\right]\left[\begin{array}{cc}
1 & d_{2} \\
0 & 1
\end{array}\right]\left[\begin{array}{cc}
1 & 0 \\
-\frac{1}{f} & 1
\end{array}\right] } \\
& \times\left[\begin{array}{cc}
1 & d_{1}+R \\
0 & 1
\end{array}\right]\left[\begin{array}{cc}
1 & 0 \\
-\frac{2}{R} & 1
\end{array}\right]\left[\begin{array}{cc}
1 & R \\
0 & 1
\end{array}\right] \\
= & {\left[\begin{array}{cc}
-1-\frac{2 d_{1}}{R}+\frac{d_{2}}{f}+\frac{d_{3}}{f} & -d_{1} \\
\frac{1}{f} & 0
\end{array}\right] }
\end{aligned}
$$

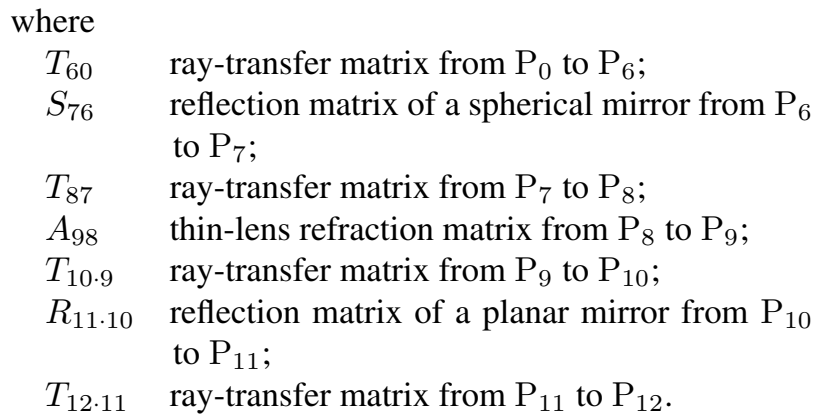

Thus, the ray at point $\mathrm{P}_{12}$ on the LCD photomask position is given by

$$
\begin{aligned}
{\left[\begin{array}{l}
y_{2} \\
\theta_{2}
\end{array}\right] } & =M_{2} *\left[\begin{array}{c}
y_{1} \\
\theta_{1}
\end{array}\right] \\
& =\left[\begin{array}{cc}
-1-\frac{2 d_{1}}{R}+\frac{d_{2}}{f}+\frac{d_{3}}{f} & -d_{1} \\
\frac{1}{f} & 0
\end{array}\right]\left[\begin{array}{l}
y_{1} \\
\theta_{1}
\end{array}\right] .
\end{aligned}
$$

If $d_{1}=\mathrm{f}$ and $y_{1}=0,(10)$ can be simplified as

$$
\left[\begin{array}{l}
y_{2} \\
\theta_{2}
\end{array}\right]=\left[\begin{array}{cc}
1-\frac{d_{2}}{f}-\frac{d_{3}}{f} & f \\
-\frac{1}{f} & 0
\end{array}\right]\left[\begin{array}{c}
0 \\
\theta_{1}
\end{array}\right] .
$$

Consequently

$$
y_{2}=f \theta_{1} \quad \theta_{2}=0 .
$$

From the results of (8) and (12), the ray angle $\theta_{2}$ on the LCD photomask is independent of the incident ray angle $\theta_{1}$ in two ray trajectories. The optical system emits parallel light upward (i.e., $\theta_{2}=0$ ) through the LCD photomask to expose and solidify the photo-curable resin.

The focal length of the biconvex lens and the radius of curvature of the spherical mirror are selected for 15 and $10 \mathrm{~cm}$ due to the machine space limitations, respectively. Because the high-power xenon lamp could generate enough convection and radiation heat to affect the LCD photomask and resin, a sunshade is placed between the biconvex lens and flat mirror to reduce the heat transfer to the LCD photomask.

After constructing the optical system, measurement of the power that spreads onto the LCD photomask is necessary. This paper uses an optical power meter for measuring the light power that spreads onto the LCD photomask. The valid light area on the LCD photomask is $10 \times 10 \mathrm{~cm}^{2}$. This paper divides the valid area into 100 equal parts. Each part is measured by the light power by using an optical power meter. The measured values are shown in Fig. 11. Based on these experimental results, the light that spreads onto the LCD photomask is determined in good uniform. The light source uses a $275-\mathrm{W}$ xenon lamp. The average light power through the LCD photomask is $1.43 \mathrm{~mW}$.

\section{SOFTWARE DESIGN}

\section{A. Bucket-Sorting Algorithm}

For most RP systems, CAD models that are described in the STL file format must be sliced into contours. An effective slicing algorithm is necessary for RP systems. A simple approach is to intersect every facet with every slicing plane. This approach is time consuming. The bucket-sorting algorithm is used in the slicing preprocessing for search speed enhancement.

The bucket-sorting algorithm divides the spatial space into $N$ subspaces. Searching for triangular data that are inside these smaller subspaces is often faster than browsing the entire space. In this paper, the split space was also called a slab, as illustrated in Fig. 12. The slabs were generated by the defined maximum acceptable thickness and by the maximum and minimum $Z$-coordinate of the facets. Each slab was defined 


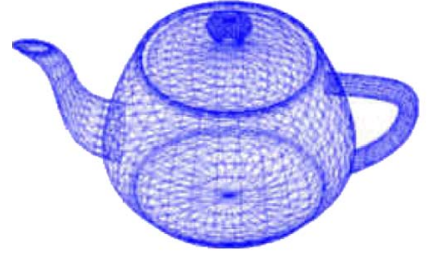

(a)

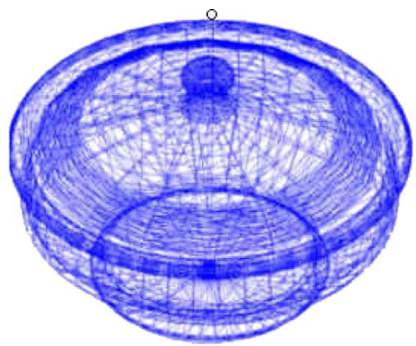

(b)
Fig. 13. (a) Facet number: 4096; height range: 56.114 in. (b) Facet number: 5192; height range: 180.718 in.

TABLE I

SLICING TIME (IN SECONDS) FOR DIFFERENT BUCKET NUMBERS [FIG. 13(a)]

\begin{tabular}{|l|c|c|c|c|c|c|}
\hline Bucket number & 1 & 5 & 10 & 20 & 30 & 40 \\
\hline Slicing time (sec) & 49.251 & 13.309 & 8.633 & 6.409 & 5.709 & 5.388 \\
\hline Time ratio & $100 \%$ & $27 \%$ & $17.5 \%$ & $13 \%$ & $11.6 \%$ & $11 \%$ \\
\hline Bucket number & 50 & 60 & 70 & 80 & 90 & 100 \\
\hline Slicing time (sec) & 5.238 & 5.257 & 5.268 & 5.287 & 5.558 & 5.598 \\
\hline Time ratio & $10.6 \%$ & $10.7 \%$ & $10.7 \%$ & $10.7 \%$ & $11.3 \%$ & $11.4 \%$ \\
\hline
\end{tabular}

TABLE II

SLICING TIME (IN SECONDS) FOR DIFFERENT BUCKET NUMBERS [FIG. 13(b)]

\begin{tabular}{|l|c|c|c|c|c|c|}
\hline Bucket number & 1 & 5 & 10 & 20 & 30 & 40 \\
\hline Slicing time (sec) & 194.28 & 50.363 & 31.826 & 23.26 & 20.29 & 18.54 \\
\hline Time ratio & $100 \%$ & $25.9 \%$ & $16.4 \%$ & $12 \%$ & $10.4 \%$ & $9.5 \%$ \\
\hline
\end{tabular}
\begin{tabular}{|l|c|c|c|c|c|c|}
\hline Bucket number & 50 & 60 & 70 & 80 & 90 & 100 \\
\hline Slicing time (sec) & 17.57 & 17.24 & 17.045 & 16.72 & 16.84 & 16.99 \\
\hline Time ratio & $9.0 \%$ & $8.9 \%$ & $8.8 \%$ & $8.6 \%$ & $8.7 \%$ & $8.8 \%$ \\
\hline
\end{tabular}

between a $Z_{\min }$ and a $Z_{\max }$ (see Fig. 12) so that, when slicing at a specific height $z$, the specific slab was the one that included $z$ within its limits $\left[\mathrm{Z}_{\min }, \mathrm{Z}_{\max }\right]$. A facet is assigned to a slab whenever one or more of its vertices fall within the slab's range. If a vertex has a $Z$ value that is exactly equal to the boundary height between two slabs, that facet is assigned to both slabs. Fig. 12 shows that the four facets (i.e., $\mathrm{F}_{4}, \mathrm{~F}_{5}, \mathrm{~F}_{6}$, and $\mathrm{F}_{8}$ ) have a common vertex $V$ and this vertex's $Z$-coordinate is equal to the boundary height $Z_{2}$ between two slabs (i.e., slabs 1 and 2). Consequently, these facets (i.e., $\mathrm{F}_{4}, \mathrm{~F}_{5}, \mathrm{~F}_{6}$, and $\mathrm{F}_{8}$ ) are assigned to both slabs 1 and 2 .

To implement the bucket-sorting algorithm, Fig. 13(a) and (b) refer to the input files. The bucket number was changed to compare the slicing time. The results (slicing time) for different bucket numbers are shown in Table I [Fig. 13(a)] and Table II [Fig. 13(b)]. If the bucket number is 1 , the bucket-sorting algorithm was not used. Based on these results, the slicing time is greatly reduced using the bucket-sorting algorithm.

The results in Tables I and II are shown in Fig. 14(a) and (b). Based on these results, the slicing time is greatly reduced by the bucket-sorting algorithm. The slicing time ratio can be reduced by nearly $25 \%$ with five buckets. In Table I, the slicing time ratio will reach the minimum value with 50 buckets. If the bucket number is greater than this certain value (i.e., 50 for Table I), the slicing time will increase. However, in Table II, the fastest slicing time will occur at 80 buckets. This optimum bucket number for slicing time is not a fixed value. It depends on the RP part's height, its facet number, and the size of the

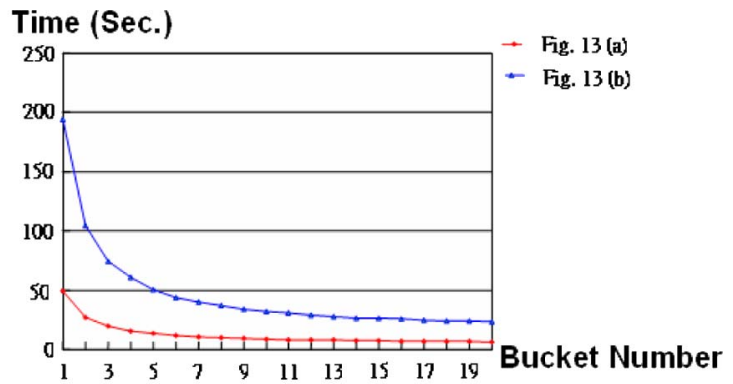

(a)

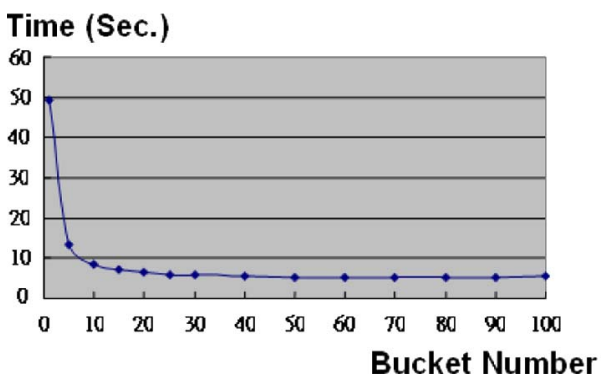

(b)

Fig. 14. (a) Slicing time for different bucket numbers. (b) Slicing time for different bucket numbers.

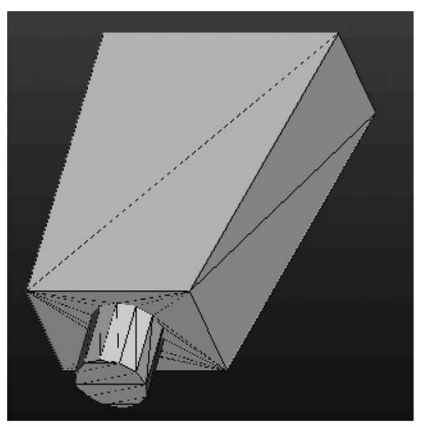

(a)

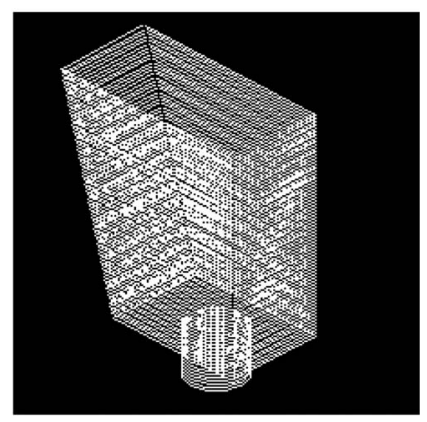

(b)
Fig. 15. (a) STL model created by Pro/Engineering CAD software. (b) STL model that was sliced using the slicing process.

facets. In general, the optimum bucket number value is $8 \sim 10$. If the bucket number is greater than 10 , the slicing time will not obviously be decreased. This means that many buckets are not useful for reducing the slicing time.

\section{B. LCD Photomask Display Algorithm}

The LCD photomask displays the cross-sectional contours of model layers, and the optical system can project the light through the white areas of the photomask. The LCD photomask display algorithm is described as follows. The program fills the inside of the contour with white color and fills the outside of the contour with black color. The light beam shines through the white areas to cure the resin. After curing one layer, the program will display the cross-sectional contour of the next layer in the LCD photomask. When all layers have been built, the program stops the RP machine, and the physical part is finished.

We used Visual Basic as the algorithm compiler. The program outputs display data to the LCD photomask to display the filled contours layer by layer. Fig. 15(a) shows an STL file that is created by Pro/Engineering 3-D CAD software. Fig. 15(b) is 

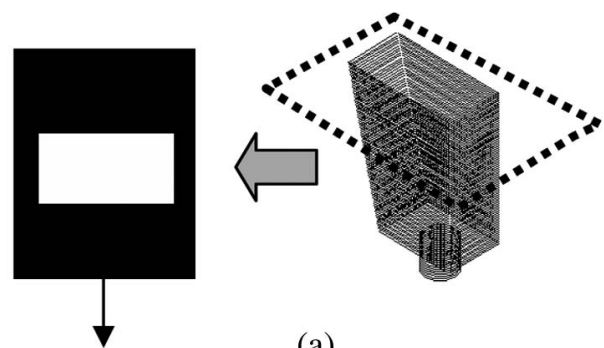

(a)

Projective display
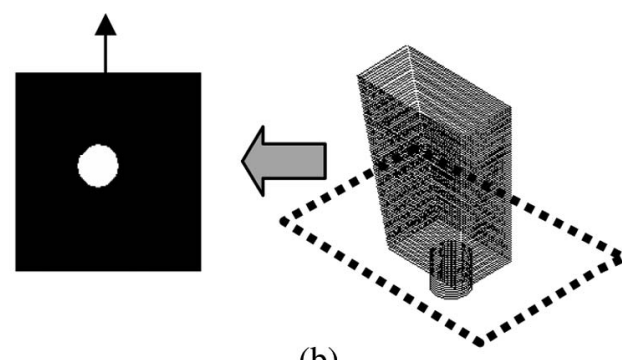

(b)

Fig. 16. LCD photomask shows the cross-sectional contours that are filled with white color.

TABLE III

EXPERIMENTAL RESULTS: EXPOSURE TIME (RELATIVE TO THE HARDENED DEPTH OF THE RESIN)

\begin{tabular}{|l|c|c|c|c|c|c|c|c|c|}
\hline & 1 & 2 & 3 & 4 & 5 & 6 & 7 & 8 & 9 \\
\hline $\begin{array}{l}\text { Exposure time } \\
\text { (sec) }\end{array}$ & 15 & 30 & 45 & 60 & 75 & 90 & 105 & 120 & $\mathbf{1 3 5}$ \\
\hline $\begin{array}{l}\text { Hardened } \\
\text { thickness (mm) }\end{array}$ & 0.010 & 0.015 & 0.030 & 0.050 & 0.06 & 0.100 & 0.150 & 0.200 & $\mathbf{0 . 2 5 4}$ \\
\hline
\end{tabular}

the result after slicing the STL file. Fig. 16(a) and (b) shows the cross-sectional contours that are filled with white color inside.

As described above, the proposed desktop manufacturing system uses the photo-curable liquid resin NAF-200N as the building material. This resin will solidify under exposure to 680-nm-wavelength light. Based on the experimental results, the relation between the exposure time and the hardened depth of the resin is illustrated in Table III. In general, the proposed system uses uniform slicing, and the layer thickness is set to $0.254 \mathrm{~mm}$ so that the exposure time for each layer is $135 \mathrm{~s}$.

\section{EXPERIMENTAL RESULTS}

To compare the machining efficiency, the FDM 2000 RP machine (from the Stratasys, Inc.) has been chosen for comparison. Although FDM 2000 is among the slowest systems, it is the only RP system that is available in our laboratory. A $100 \mathrm{~mm} \times 100 \mathrm{~mm} \times 1 \mathrm{~mm}$ thin plate is manufactured by the FDM 2000 and the proposed RP system. After finishing the slicing process, the tool path for the FDM 2000 RP machine is shown in Fig. 17(a), and the LCD photomask display of the proposed RP system is shown in Fig. 17(b). The manufacturing time for building one layer is $582 \mathrm{~s}$ in FDM 2000. However, the manufacturing time for building one layer is $135 \mathrm{~s}$ in the proposed RP system. The machining efficiency of the proposed system is better than the FDM 2000 RP system. The accuracy of the proposed RP system is $0.4 \mathrm{~mm}$ (i.e., $0.015 \mathrm{in}$ ), which is sufficient for real applications.

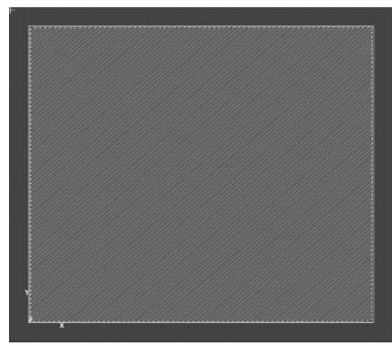

(a)

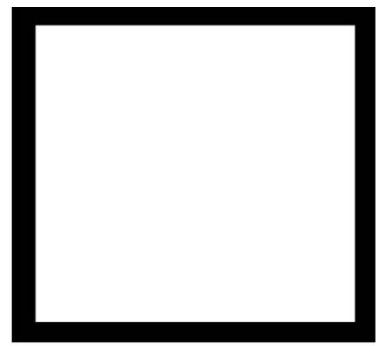

(b)
Fig. 17. (a) Tool path for the FDM 2000 RP machine. (b) LCD photomask display of the proposed RP system.

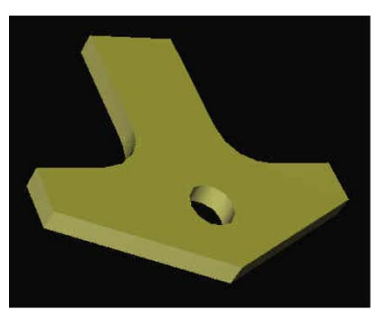

(a)

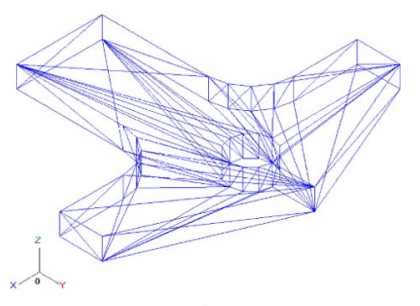

(b)
Fig. 18. (a) Three-dimensional CAD model of Case 1. (b) STL model of Case 1.

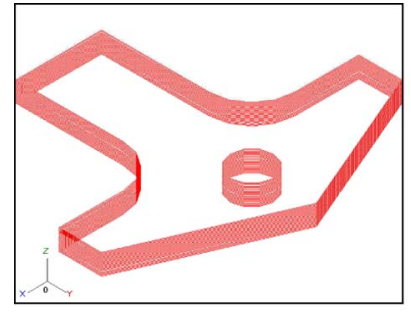

(a)

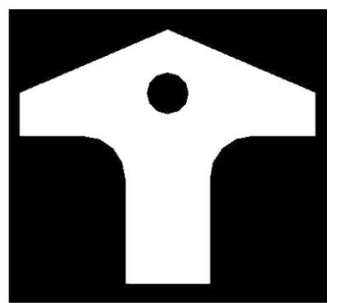

(b)
Fig. 19. (a) Illustration of the sliced model. (b) Cross-sectional contour that is displayed in the LCD photomask.
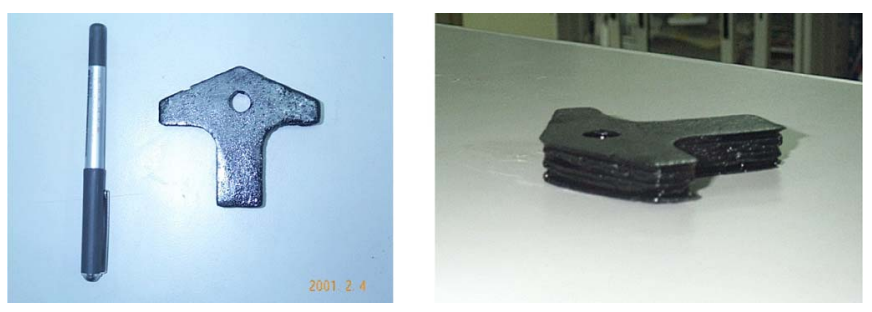

Fig. 20. RP part of Case 1, which was built by the proposed desktop manufacturing system.

\section{A. Case 1}

In Case 1, the 3-D CAD model is illustrated in Fig. 18(a), and the STL model is shown in Fig. 18(b). The RP software first reads the STL file and proceeds with the slicing process. The sliced model is shown in Fig. 19(a).

The LCD photomask displays the cross-sectional contour, and the proposed desktop manufacturing system builds the physical model. The cross-sectional contour that is displayed in the LCD photomask is illustrated in Fig. 19(b). The physical part is built, layer by layer, using the proposed system. The finished RP part for Case 1 is shown in Fig. 20. 


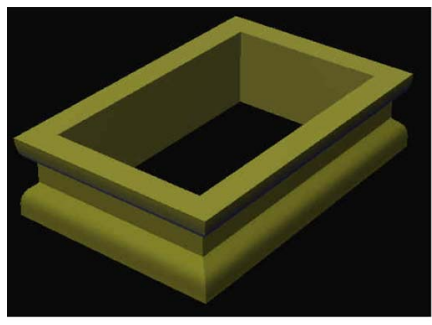

(a)

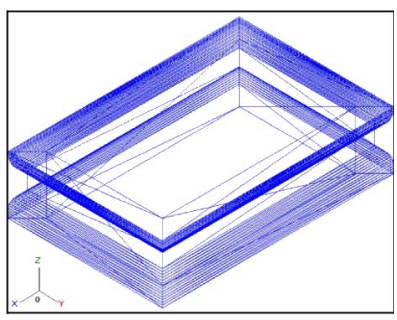

(b)
Fig. 21. (a) Three-dimensional CAD model of Case 2. (b) STL file of Case 2.

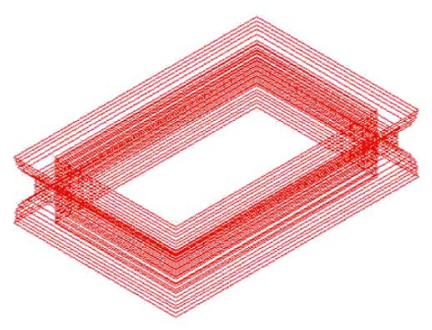

(a)

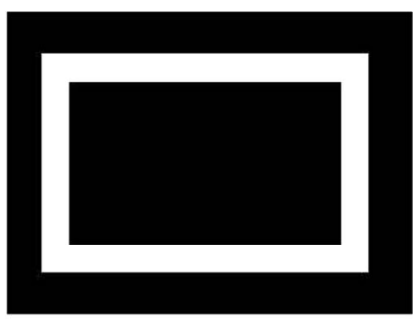

(b)
Fig. 22. (a) Sliced model of Case 2. (b) Cross-sectional contour in the LCD photomask.

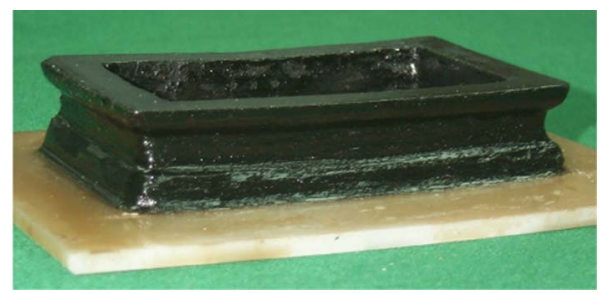

Fig. 23. RP part of Case 2, which was built by the proposed desktop manufacturing system.

TABLE IV

EXPERIMENTAL RESULTS OF CASES 1 AND 2

\begin{tabular}{|c|c|c|c|c|}
\hline & Layer number & Layer thickne ss & $\begin{array}{c}\text { Curing time of } \\
\text { each layer }\end{array}$ & Total curing time \\
\hline CASE 1 & 49 & $0.254 \mathrm{~mm}$ & $135 \mathrm{sec}$ & $110.25 \mathrm{~min}$ \\
\hline CASE 2 & 60 & $0.254 \mathrm{~mm}$ & $135 \mathrm{sec}$ & $135 \mathrm{~min}$ \\
\hline
\end{tabular}

\section{B. Case 2}

In Case 2, the 3-D CAD model is illustrated in Fig. 21(a), and the STL model is shown in Fig. 21(b). After slicing the STL file, the sliced model is illustrated in Fig. 22(a), and the LCD photomask cross-sectional contour is illustrated in Fig. 22(b). The physical part that was built using the proposed system is shown in Fig. 23.

The experimental results of Cases 1 and 2 are also shown in Table IV.

\section{CONCLUSION}

Desktop manufacturing systems have widely been known as being capable of fabricating 3-D objects with complex geometric shapes. The purpose of this paper has been to develop an LCD-photomask-based desktop manufacturing system.
The main features of the proposed system are described as follows.

1) The LCD photomask is connected to the computer to allow for quickly changing the cross-sectional display of each layer.

2) The software design includes a slicing algorithm, an LCD photomask display, a process-user interface, and a motion control program. The bucket-sorting algorithm is used in the slicing preprocessing for search speed enhancement. The slicing time ratio can be reduced to nearly $25 \%$ with five buckets.

3) The hardware configuration of this architecture includes an LCD photomask, an optical system, a $z$-axis elevator, and a PC-based control system.

4) The matrix optics is used to design the optical system.

5) The experimental results show that the proposed desktop manufacturing system can produce RP parts with good machining efficiency, but the surface roughness should further be improved.

6) The proposed desktop manufacturing system has the advantages of low cost, compactness, speed and accuracy, and no additional support requirement, providing a valuable addition to the working office environment for designers at all levels and in all locations.

\section{REFERENCES}

[1] N. Puntambenker, A. Jablokow, and H. Sommer, "A unified review of 3D model generation for reverse engineering," Comput. Integr. Manuf., vol. 7, no. 4, pp. 259-268, 1994.

[2] T. Soboh, J. Owen, C. Jaynes, M. Dekhil, and T. Henderson, "Industrial inspection and reverse engineering," in Proc. IEEE CAD-Based Vis., 1995, pp. $228-235$.

[3] C. K. Chua, "Three-dimensional rapid prototyping technologies and key development areas," Comput. Control Eng. J., vol. 5, no. 4, pp. 200-206, Aug. 1994.

[4] R. C. Luo and Y. Ma, "A slicing algorithm for rapid prototyping and manufacturing," in Proc. IEEE Int. Conf. Robot. Autom., 1995, pp. 2841-2846.

[5] P. Kulkarni, A. Marsan, and D. Dutta, "A review of process planning techniques in layered manufacturing," Rapid Prototyping J., vol. 6, no. 1, pp. 18-35, 2000.

[6] [Online]. Available: http://www.rpm3d.com/

[7] [Online] Available: http://www.2objet.com/

[8] [Online]. Available: http://www.envisiontec.de/index.htm

[9] [Online]. Available: http://www.3dsystems.com/

[10] [Online]. Available: http://www.stratasys.com/

[11] [Online]. Available: http://www.cubital.com/

[12] R. C. Luo, J. H. Tzou, and W. Z. Lee, "The development of LCD panel display based rapid prototyping system for advanced manufacturing," in Proc. IEEE Int. Conf. Robot. Autom., San Francisco, CA, Apr. 24-28, 2000, vol. 4, pp. 3083-3088.

[13] S. C. Ventura, S. C. Narang, S. Sharma, J. Stotts, C. Liu, S. Liu, L.-H. Ho, D. Annavajjula, S. J. Lombardi, A. Hardy, M. Mangaudis, E. Chen, and L. Groseclose, "A new SFF process for functional ceramic components," in Proc. Solid Freeform Fabrication, 1996, pp. 327-334.

[14] J. S. Young, S. R. Fox, and K. S. Anseth, "A novel device for producing three-dimensional objects," J. Manuf. Sci. Eng., vol. 121, no. 3, pp. 474477, Aug. 1999.

[15] S. Monneret, H. Le Gall, V. Bade, F. Devaux, A. Mosset, and E. Lantz, "Dynamic UV microstereolithography," Eur. Phys. J. Appl. Phys., vol. 20, no. 3, pp. 213-218, Dec. 2002.

[16] Y.-M. Huang and C.-P. Jiang, "Numerical analysis of a mask type stereolithography process using a dynamic finite element method," Int. J. Adv. Manuf. Technol., vol. 21, no. 9, pp. 649-655, Jun. 2003.

[17] C.-P. Jiang, J.-Y. Jeng, Y.-M. Huang, and M.-J. Tsai, "Development of masked photo-polymerization rapid prototyping system using liquid crystal display panel," J. Chin. Inst. Ind. Eng., vol. 22, no. 1, pp. 76-81, 2005.

[18] E. Hecht, OPTICS, 4th ed. San Francisco, CA: Addison-Wesley, 2002.

[19] B. E. A. Saleh and M. C. Teich, Fundamentals of Photonics. Hoboken, NJ: Wiley, 1991. 


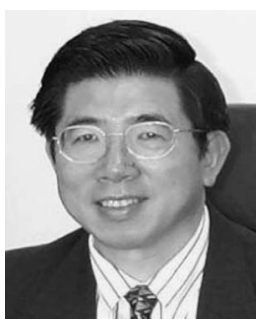

Ren C. Luo (M'82-SM'87-F'92) received the Ph.D. degree from the Technische Universität Berlin, Berlin, Germany.

He was a Research Engineer with Waldrich Siegen, Burbach, Germany, and was a Scientific Research Staff with the Fraunhofer Institute for Production and Design, Berlin. He was a Scientific Research Staff with the Institute for Measurement and Control Engineering, Berlin, contributing on the design of various sensors-integrated control systems. He was an Assistant Professor of electrical engineering and computer science with the University of Illinois, Chicago, teaching and researching on sensor-based robots and flexible automation. He later joined the Department of Electrical and Computer Engineering as a Professor and became the founding Director of the Center for Robotics and Intelligent Machines, North Carolina State University, Raleigh. He was the Toshiba Chair Professor of Electrical Engineering with the Institute of Industrial Science, University of Tokyo, Tokyo, Japan. For six years, he has been the Dean of the College of Engineering, National Chung Cheng University, Chia-Yi, Taiwan, R.O.C., where he was also the President from 2001 to 2007 . He is currently a Professor with the Department of Electrical Engineering, National Taiwan University, Taipei, Taiwan. He has published more than 300 refereed publications and is the holder of several patents. His research contributions include sensorcontrolled intelligent robotics systems, multisensor fusion and integration, visual servocontrol systems, rapid prototyping and advanced automation systems, microtechnologies, and nanotechnologies.

Prof. Luo is a Fellow of the Institution of Engineering and Technology and the President of the Robotics Society of Taiwan. For five years, he was the Editor-in-Chief of the IEEE/ASME TRANSACTIONS ON MECHATRONICS. For more than ten times, he has served as the General Chair of IEEE and other international conferences. He received the IEEE Eugean Mittleman Outstanding Research Achievement Award, the Alcoa Foundation Distinguished Engineering Research Award from North Carolina State University, the Outstanding Achievement Award from Banki Donat University, Hungary, the TECO Outstanding Science and Technology Research Achievement Award, the National Science Council Outstanding Research Awards for seven consecutive years, the Automation Engineering Medal Award from the Institute of Automation Engineers, and Outstanding Engineering Professor Award from the Chinese Institute of Engineers. He and his students have twice received the First Place (Championship) in the American Association of Artificial Intelligence International Robots Competition in 1993 and 1995, respectively, and the International Student Experimental Hands-On Project Competition via Internet on Intelligent Mechatronics and Automation in 2004. He also received the Excellent Paper and Research Result Competition Award from the Institute of Information and Computing Machinery of Taiwan.

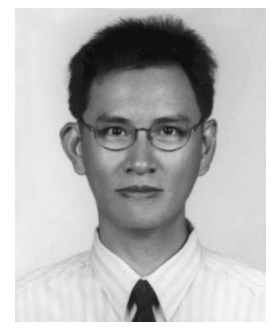

Jyh Hwa Tzou received the B.S. degree in mechanical engineering from the National Cheng Kung University, Tainan, Taiwan, R.O.C., in 1986, the M.S. degree in mechanical engineering from the National Sun Yat-Sen University, Kaohsiung, Taiwan, in 1988, and the Ph.D. degree in electrical engineering from the National Chung Cheng University, Chia-Yi, Taiwan, in 2005.

$\mathrm{He}$ is currently an Associate Professor with the Department of Mechanical Engineering, Wu-Feng Institute of Technology, Chia-Yi. His research interests include rapid prototyping, rapid tooling, intelligent robotic systems, and mechatronic systems. 